\title{
BRCA1 Tumor Suppression Depends on BRCT Phosphoprotein Binding, But Not Its E3 Ligase Activity
}

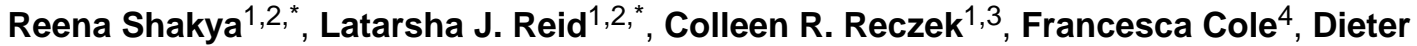 \\ Egli $^{5}$, Chyuan-Sheng Lin ${ }^{2}$, Dirk G. deRooij ${ }^{6}$, Steffen Hirsch ${ }^{1,2}$, Kandasamy Ravi ${ }^{7}$, James B. \\ Hicks $^{7}$, Matthias Szabolcs ${ }^{2,8}$, Maria Jasin ${ }^{4}$, Richard Baer ${ }^{1,2,8, \dagger}$, and Thomas Ludwig ${ }^{1,2,8, \dagger}$ \\ ${ }^{1}$ Institute for Cancer Genetics, Columbia University, New York, NY 10032, USA ${ }^{2}$ Department of \\ Pathology and Cell Biology, Columbia University, New York, NY 10032, USA ${ }^{3}$ Department of \\ Nutritional and Metabolic Biology, Columbia University, New York, NY 10032, USA \\ ${ }^{4}$ Developmental Biology Program, Memorial Sloan-Kettering Cancer Center, New York, NY \\ 10021, USA ${ }^{5}$ New York Stem Cell Foundation (NYSCF), New York, NY 10032, USA ${ }^{6}$ Center for \\ Reproductive Medicine, Amsterdam Medical Center, University of Amsterdam, 1105 AZ \\ Amsterdam, Netherlands ${ }^{7}$ Cold Spring Harbor Laboratory, Cold Spring Harbor, NY 11724, USA \\ ${ }^{8}$ Herbert Irving Comprehensive Cancer Center, Columbia University, New York, NY 10032, USA
}

\section{Abstract}

Germline mutations of the breast cancer 1 (BRCA1) gene are a major cause of familial breast and ovarian cancer. The BRCA1 protein displays E3 ubiquitin ligase activity, and this enzymatic function is thought to be required for tumor suppression. To test this hypothesis, we generated mice that express an enzymatically defective Brca1. We found that this mutant Brcal prevents tumor formation to the same degree as does wild-type Brca1 in three different genetically engineered mouse (GEM) models of cancer. In contrast, a mutation that ablates phosphoprotein recognition by the BRCA C terminus (BRCT) domains of BRCA1 elicits tumors in each of the three GEM models. Thus, BRCT phosphoprotein recognition, but not the E3 ligase activity, is required for BRCA1 tumor suppression.

\begin{abstract}
Germline mutations of the breast cancer 1 (BRCAl) tumor suppressor are a common cause of hereditary breast and ovarian cancer. The BRCA1 protein harbors an N-terminal RING motif characteristic of many ubiquitin E3 ligases and two BRCA C terminus (BRCT) motifs that form a phosphoprotein recognition domain (1-4). BRCA1 interacts with BRCA1associated RING domain protein 1 (BARD1) to form a potent E3 ligase $(5,6)$ that is thought to regulate multiple pathways, including those responsible for tumor suppression (1-4). To test whether the E3 ligase activity of BRCA1 is essential for tumor suppression, we examined mice that express Brca1 ${ }^{\mathrm{FH}-\mathrm{I} 26 \mathrm{~A}}$, an enzymatically defective protein with a missense mutation (I26A) in the RING domain that allows assembly of the BRCA1/BARD1 heterodimer but abrogates its E3 ligase activity $(7,8)$. (Single-letter abbreviations for the amino acid residues are as follows: A, Ala; C, Cys; D, Asp; E, Glu; F, Phe; G, Gly; H, His; I, Ile; K, Lys; L, Leu; M, Met; N, Asn; P, Pro; Q, Gln; R, Arg; S, Ser; T, Thr; V, Val; W,
\end{abstract}

\footnotetext{
${ }_{*}^{\dagger}$ To whom correspondence should be addressed. t154@ columbia.edu (T.L.); rb670@ columbia.edu (R.B.).

*These authors contributed equally to this work.

Supporting Online Material

www.sciencemag.org/cgi/content/full/334/6055/525/DC1

Materials and Methods

Figs. S1 to S6

References
} 
Trp; and Y, Tyr. In the mutants, other amino acids were substituted at certain locations; for example, I26A indicates that isoleucine at position 26 was replaced by alanine.)

$\mathrm{BrCal}^{\mathrm{FH}-\mathrm{I} 26 \mathrm{~A} /+}$ and $\mathrm{BrCal}^{\mathrm{FH}-\mathrm{WT} /+}$ mice were generated from isogenic embryonic stem (ES) cells that express either Brca $1^{\mathrm{FH}-\mathrm{I} 26 \mathrm{~A}}$ or the corresponding wild-type Brca ${ }^{\mathrm{FH}-\mathrm{WT}}$ protein (9) and bred to homozygosity. Brcal ${ }^{\text {FH-I26A/FH-I26A }}$ pups were born at the expected Mendelian ratio and survived to adulthood. Because Brca1-null mice invariably undergo embryonic lethality $(10,11)$, the viability of $B r c a 1^{F H-I 26 A / F H-I 26 A}$ mice indicates that E3 ligase activity is not essential for all BRCA1 functions. Nonetheless, Brcal ${ }^{F H-I 26 A / F H-I 26 A}$, but not Brcal ${ }^{F H-W T / F H-W T}$, males are sterile and have smaller testes, and their seminiferous tubules lack elongated spermatids and spermatozoa (fig. S1), which suggests a block in spermatogenesis. Apart from male sterility and a modest decrease in adult body weight (5 to 10\%), Brcal ${ }^{F H-I 26 A / F H-I 26 A}$ mice appear indistinguishable from their Brcal ${ }^{F H-W T / F H-W T}$ littermates. Moreover, analyses of Brcal ${ }^{F H-I 26 A / F H-I 26 A}$ mouse embryonic fibroblasts (MEFs) revealed that the I26A mutation had no measurable effect on cellular proliferation, chromosomal stability, senescence induction, centrosome number, spindle formation, resistance to genotoxic stress, or ubiquitin foci formation at sites of DNA damage - unlike the hypomorphic Brcal lesions in MEFs of Brcal ${ }^{\text {tr/tr }}$ (12) and Brcal ${ }^{\Delta 11 / \Delta 11}$ mice (13), which are known to disrupt tumor suppression (fig. S2).

To evaluate whether the E3 ligase activity affects tumor suppression, we initially used a mouse model of pancreatic cancer in which the $P d x l$-cre transgene triggers Kras ${ }^{\mathrm{G} 12 \mathrm{D}}$ and $\mathrm{p} 53^{\mathrm{R} 172 \mathrm{H}}$ expression in pancreatic progenitor cells (14). To test whether Brcal suppresses formation of these tumors, we generated Pdxl-cre animals carrying Kras ${ }^{L S L-G 12 D}$ (14) together with conditional-null Brcalflex2 (15) and/or $p 53^{f l e x 7}$ (16) alleles. Although doublemutant $K r a s{ }^{L S L-G 12 D} / p 53^{f l e x} 7 / f l e x 7 / P d x 1$-cre mice succumbed to pancreatic tumors with an average latency $\left(T_{50}\right)$ of 68 days, tumor latency was dramatically reduced in triple-mutant

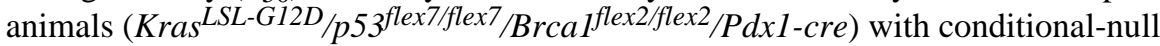
Brcal flex $2^{f}\left(T_{50}=40\right.$ days; $\left.P<0.0001\right)$, indicating that wild-type Brca1 suppresses pancreatic tumor development (Fig. 1A). In contrast, triple-mutant animals (Kras ${ }^{L S L-G 12 D /}$ p5 $3^{\text {flex7/flex7 } / B r c a l}$ flex2/FH-I26A/Pdxl-cre) expressing Brcal $^{\text {FH-I26A }}$ developed pancreatic tumors with a similar latency $\left(T_{50}=65\right.$ days $)$ as that of double-mutant Kras ${ }^{L S L}-G 12 D /$

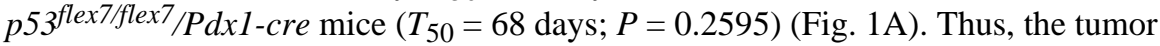
suppression potential of enzymatically inactive Brcal in the pancreas is indistinguishable from that of wild-type Brca1.

We next applied a mouse model of familial breast cancer in which the Wap ${ }^{\text {cre }}$ gene elicits mammary-specific inactivation of the conditional-null Brcal flex2 allele (15). However,

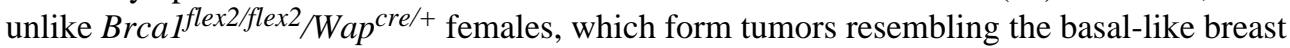
carcinomas of human BRCA1 mutation carriers (15), all mice expressing enzymatically inactive Brca1 (Brcal ${ }^{f l e x 2 / F H-I 26 A} /$ Wap $^{\text {cre/+ }}$ ) remained tumor-free over the 24-month observation period (Fig. 1B).

We also monitored three cohorts of Wap $^{\text {cre/+ }}$ mice sensitized for tumor development by a conditional $p 53$ mutation $\left(p 53^{L S L-R 270 H}\right.$ ). Whereas mammary tumors developed in control $p 53^{L S L-R 270 H /+} / \mathrm{Wap}^{\mathrm{cre} /+}$ females with an average latency of 380 days, which is consistent with previous studies (17), tumor formation was accelerated $\left(T_{50}=308\right.$ days) by conditional Brca1 inactivation in Brcalflex2/flex2 $/ p 53^{L S L-R 270 H /+} /$ Wap $^{\text {cre/+ }}$ females $(P<0.0001)$ (Fig.

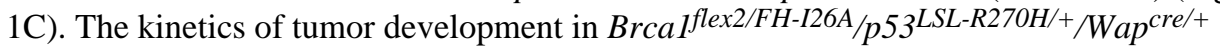
females was indistinguishable from that of control $p 53^{L S L}-R 270 H /+/ \mathrm{Wap}^{\text {cre/+ }}$ females $(P=$ 0.7502) and significantly slower than that of Brcal flex2/flex $2 / p 53^{L S L-R 270 H /+} / \mathrm{Wap}^{\mathrm{cre} /+}$ females $(P<0.0001)$ (Fig. 1C). Moreover, representative oligonucleotide microarray analysis (18) revealed a "simplex" pattern of genomic copy number variation in

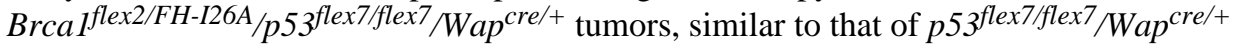


tumors but distinct from the "complex sawtooth" pattern of Brcalflex2/flex2/p53flex7/flex7/ Wap ${ }^{\text {cre/+ }}$ tumors (fig. S3). Thus, mammary-specific loss of Brca1 enzymatic activity does not promote basal-like breast carcinoma in a manner analogous to complete Brca1 inactivation.

Although mice lacking Brcal enzymatic activity (Brcal ${ }^{\text {FH-I26A/FH-I26A }}$ and Brcal $^{\text {FH-I26A/- }}$ ) are viable, some (18.6\%) died of tumors at a very advanced age (Fig. 1D). However, the frequency and kinetics of spontaneous tumor formation in the mutant mice were comparable with those of Brcal $^{F H-W T / F H-W T}$ control animals $(P=0.5197)$ and significantly lower than those of $\mathrm{Brcal}^{\mathrm{tr} / \mathrm{tr}}$ mice $(P<0.0001)(12)$. Thus, the E3 ligase activity of BRCA1 is dispensable for tumor suppression in each of the three GEM cancer models.

The BRCT motifs of BRCA1 form a phospho-recognition domain that preferentially binds the phosphorylated isoforms of repair proteins Abraxas/CCDC98, BACH1/FancJ, and CtIP $(1,2)$. Because most tumor-associated BRCA1 alleles have frameshift/nonsense mutations that eliminate one or both BRCT motifs, BRCT phospho-recognition may be critical for tumor suppression. Indeed, in some families breast cancer susceptibility can be ascribed to missense mutations that cause a single amino acid substitution (for example, S1655F) that disrupts the interaction between the BRCT domain and its cognate phospho-ligands. Structural studies show that BRCA1 residue S1655 donates a hydrogen bond to the phosphate group of these phospho-ligands, and that mutation of this residue disrupts their interaction with BRCA1 (19-23). To determine whether BRCT phospho-recognition is required for genome stability and tumor suppression, we mutated the corresponding mouse residue $(\mathrm{S} 1598 \mathrm{~F})$ to generate heterozygous $\left(\right.$ Brcal $\left.^{\text {S1598F/+ }}\right)$ and homozygous

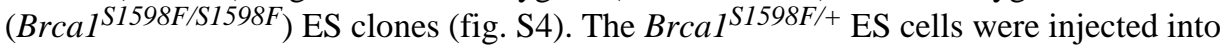
blastocysts to derive germline chimeras, and heterozygous animals were then intercrossed to produce homozygous Brcal ${ }^{S 1598 F / S 1598 F}$ offspring, which appeared at the expected Mendelian ratio. Apart from male sterility, these mice developed normally and provided a

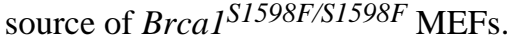

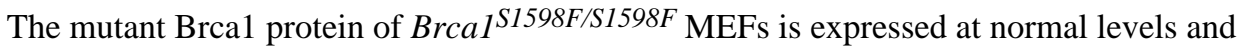
fails to bind Bach1/FancJ (Fig. 2A). Brcal ${ }^{S 1598 F / S 1598 F}$ ES cells are hypersensitive to genotoxic stress (Fig. 2B) and defective for homology-directed DNA repair (Fig. 2C). In addition, Brcal ${ }^{S 1598 F / S 1598 F}$ MEFs display proliferation defects, chromosomal instability, centrosome amplification, and diminished recruitment of repair proteins to sites of DNA damage (figs. S5 and S6). Thus, the S1598F mutation disrupts BRCA1 function in the DNA damage response. To evaluate its effect on tumor suppression, we monitored an experimental cohort of Brcal flex $2 / S 1598 F / p 53^{L S L-R 270 H /+} /$ Wap $^{\text {cre/+ }}$ females and control cohorts of the $p 53^{\text {LSL-R270H/+} / \text { Wap }^{\text {cre/+}} \text {, Brcalflex2/flex2/Wap }}{ }^{\text {cre/+}}$, and Brcal flex2/flex2/ p53 $3^{L S L-R 270 H /+} /$ Wap $^{\text {cre/+ }}$ females described above (Fig. 1, B and C). As shown in Fig. 3A, mammary tumorigenesis was accelerated in experimental Brcal flex2/S1598F/p53 $3^{L S L-R 270 H /+}$ / Wap $^{\text {cre/+ }}$ mice $\left(T_{50}=308\right.$ days $)$ relative to $p 53^{L S L-R 270 H /+} /$ Wap $^{\text {cre/++}}$ animals $\left(T_{50}=380\right.$ days; $P<0.0001)$, and the shortened latency was indistinguishable from that of Brcal ${ }^{\text {flex } 2 / f l e x} 2 / p 53^{L S L-R 270 H /+} /$ Wap $^{\text {cre/+ }}$ animals $\left(T_{50}=309\right.$ days; $\left.P=0.4543\right)$. Thus, the $\mathrm{S} 1598 \mathrm{~F}$ mutation impairs mammary tumor suppression to a comparable degree as does complete Brcal inactivation. Pancreatic tumor formation in mice expressing Brcal $1598 \mathrm{~F}$

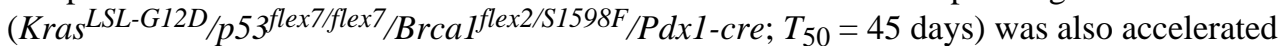
relative to those expressing wild-type Brca1 (Kras ${ }^{L S L-G 12 D} / p_{53}$ flex $7 / f l e x 7 / \mathrm{Pdx} 1$-cre mice; $T_{50}$ $=68$ days $P<0.0001)$ (Fig. $3 \mathrm{~B})$ and comparable with conditional-null Brca1 mice

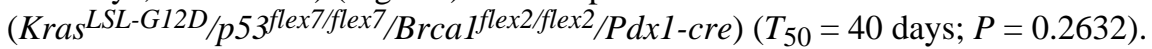
Furthermore, homozygous Brcal ${ }^{S 1598 F / S 1598 F}$ mice were highly tumor-prone (49 out of 72; $68.1 \%$ ) relative to control animals $(P<0.0001)$ (Fig. 3C). Thus, BRCT phospho-recognition is critical for BRCA1-mediated tumor suppression in all three GEM cancer models. 
BRCA1 is thought to regulate diverse cellular processes by ubiquitinating multiple protein substrates (1-4). Indeed, on the basis of the same mutation (I26A) used here the E3 ligase activity has been implicated in BRCA1 control of centrosome duplication (24), checkpoint activation (25), mitotic spindle assembly (26), and tumor cell motility (27). In light of these observations, the viability of mice lacking BRCA1 enzymatic activity was unexpected. Because mice homozygous for null Brcal mutations undergo embryonic lethality $(10,11)$, our results suggest that the E3 ligase activity of BRCA1 is dispensable for much of normal development. Thus, many of the known and unknown functions of BRCA1 may be mediated independent of its ability to catalyze ubiquitination.

From a medical standpoint, the key function of BRCA1 is its ability to suppress tumor formation in breast and ovarian tissues. Like BRCA1, many other oncoproteins and tumor suppressors possess enzymatic activity. In most cases, the enzymatic function has proven to be integral to the oncogenic process, and in some cases it can be targeted for therapeutic gain, as illustrated by the clinical success of certain tyrosine kinase inhibitors. However, because enzymatically inert Brca1 is sufficient to suppress tumor formation in several settings (Fig. 1), including a model of human basal-like breast cancer, the E3 ligase activity of BRCA1 appears to be dispensable for tumor suppression.

A role for the E3 ligase activity in BRCA1 tumor suppression was predicated partly on the fact that this activity is ablated by certain tumor-associated lesions of the RING domain, such as the $\mathrm{C} 61 \mathrm{G}$ and $\mathrm{C} 64 \mathrm{G}$ missense mutations (6). However, these mutations also disrupt the interaction between BRCA1 and its partner protein BARD1 (5). Because mammaryspecific inactivation of either Brcal or Bardl elicits breast tumors with the same basal-like phenotype (15), the tumor suppression activity of BRCA1 is probably mediated by the BRCA1/BARD1 heterodimer. Unlike the tumorigenic C61G and C64G mutations, the synthetic I26A mutation used in this study specifically ablates the enzymatic activity of BRCA1 but allows proper assembly of the BRCA1/BARD1 heterodimer (7). Thus, the tumorigenic RING mutations are likely to compromise BRCA1-mediated tumor suppression primarily by impairing BRCA1/BARD1 heterodimerization.

The mouse model of hereditary breast cancer has provided meaningful insights into the molecular mechanisms of BRCA1-mediated tumor suppression. First, the identical pattern of breast carcinogenesis in conditional Brcal-, Bard1-, and Brcal/Bardl-null mice implies that tumor suppression is dependent on the BRCA1/BARD1 heterodimer (15). Second, as shown here tumor suppression does not require the E3 ligase activity of BRCA1/BARD1. Third, our data also suggest that the ability of the BRCT domain to bind its phospho-ligands is critical for BRCA1 tumor suppression. In light of this result, it is noteworthy that BRCA1/ BARD1 forms distinct protein complexes (A, B, and C) based on BRCT-mediated interactions with three different repair proteins (Abraxas/CCDC98, BACH1/FancJ, and CtIP) $(1,2)$. Because tumor suppression appears to be dependent on BRCA1 association with one or more of these BRCT phospho-ligands (and/or others yet to be discovered), these interactions may provide valuable targets for therapeutic intervention.

\section{Supplementary Material}

Refer to Web version on PubMed Central for supplementary material.

\section{Acknowledgments}

We thank X. Sun for technical assistance, V. Murty for advice, M. Wigler for discussions and encouragement, and NYSCF for access to the confocal microscope. This work was supported by NIH grants R01-CA137023 (R.B. and T.L.), P01-CA97403 (R.B. and T.L.), and R01-HD40916 (M.J.). R.S. was supported by a Susan G. Komen Breast Cancer fellowship, L.J.R. by a Kirschstein National Research Service Award fellowship (F31-CA132626), C.R.R. 
by fellowships from the National Cancer Institute (T32-CA09503) and U.S. Department of Defense (DOD) (BC083089), and F.C. by a Kirschstein National Research Service Award fellowship (F32-HD51392). K.R. and J.B.H. were supported by grants to M. Wigler and J.B.H. from DOD (W81XWH04-1-0477) and the Breast Cancer Research Foundation. Microarray data have been deposited in the National Center for Biotechnology Information's Gene Expression Omnibus (GEO) with GEO Series accession number GSE31673.

\section{References and Notes}

1. Moynahan ME, Jasin M. Nat Rev Mol Cell Biol. 2010; 11:196. [PubMed: 20177395]

2. Huen MS, Sy SM, Chen J. Nat Rev Mol Cell Biol. 2010; 11:138. [PubMed: 20029420]

3. Wu W, Koike A, Takeshita T, Ohta T. Cell Div. 2008; 3:1. [PubMed: 18179693]

4. Baer R, Ludwig T. Curr Opin Genet Dev. 2002; 12:86. [PubMed: 11790560]

5. Wu LC, et al. Nat Genet. 1996; 14:430. [PubMed: 8944023]

6. Hashizume R, et al. J Biol Chem. 2001; 276:14537. [PubMed: 11278247]

7. Brzovic PS, et al. Proc Natl Acad Sci U S A. 2003; 100:5646. [PubMed: 12732733]

8. Christensen DE, Brzovic PS, Klevit RE. Nat Struct Mol Biol. 2007; 14:941. [PubMed: 17873885]

9. Reid LJ, et al. Proc Natl Acad Sci U S A. 2008; 105:20876. [PubMed: 19088202]

10. Hakem R, et al. Cell. 1996; 85:1009. [PubMed: 8674108]

11. Ludwig T, Chapman DL, Papaioannou VE, Efstratiadis A. Genes Dev. 1997; 11:1226. [PubMed: 9171368]

12. Ludwig T, Fisher P, Ganesan S, Efstratiadis A. Genes Dev. 2001; 15:1188. [PubMed: 11358863]

13. Xu X, et al. Mol Cell. 1999; 3:389. [PubMed: 10198641]

14. Hingorani SR, et al. Cancer Cell. 2005; 7:469. [PubMed: 15894267]

15. Shakya R, et al. Proc Natl Acad Sci U S A. 2008; 105:7040. [PubMed: 18443292]

16. Chen Z, et al. Nature. 2005; 436:725. [PubMed: 16079851]

17. Wijnhoven SW, et al. Cancer Res. 2005; 65:8166. [PubMed: 16166291]

18. Hicks J, et al. Genome Res. 2006; 16:1465. [PubMed: 17142309]

19. Botuyan MV, et al. Structure. 2004; 12:1137. [PubMed: 15242590]

20. Clapperton JA, et al. Nat Struct Mol Biol. 2004; 11:512. [PubMed: 15133502]

21. Shiozaki EN, Gu L, Yan N, Shi Y. Mol Cell. 2004; 14:405. [PubMed: 15125843]

22. Varma AK, Brown RS, Birrane G, Ladias JA. Biochemistry. 2005; 44:10941. [PubMed: 16101277]

23. Williams RS, Lee MS, Hau DD, Glover JN. Nat Struct Mol Biol. 2004; 11:519. [PubMed: 15133503]

24. Sankaran S, Starita LM, Simons AM, Parvin JD. Cancer Res. 2006; 66:4100. [PubMed: 16618730]

25. Yu X, Fu S, Lai M, Baer R, Chen J. Genes Dev. 2006; 20:1721. [PubMed: 16818604]

26. Joukov V, et al. Cell. 2006; 127:539. [PubMed: 17081976]

27. Coene ED, et al. J Cell Biol. 2011; 192:497. [PubMed: 21282464]

28. Moynahan ME, Chiu JW, Koller BH, Jasin M. Mol Cell. 1999; 4:511. [PubMed: 10549283] 

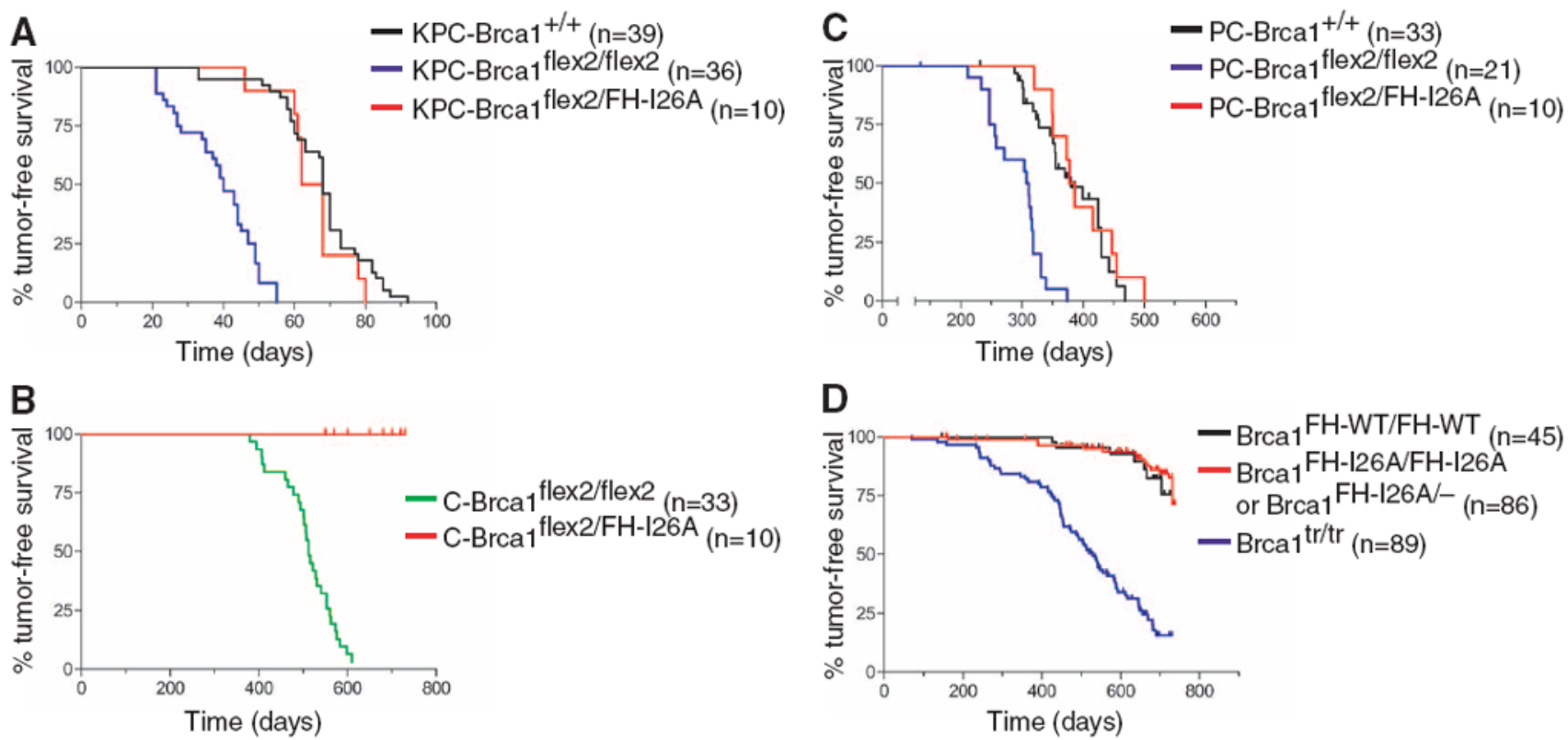

Fig. 1.

The enzymatic activity of Brca1 is dispensable for tumor suppression. (A) Kaplan-Meier

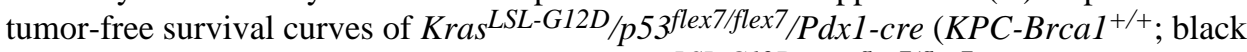
curve; $T_{50}=68$ days) mice compared with $\mathrm{Kras}^{L S L-G 12 D} / \mathrm{p}^{2} 3^{\text {flex } 7 / f l e x 7} / \mathrm{Pdxl}$-crel Brcal ${ }^{\text {flex2/flex2 }}$ (KPC-Brcal ${ }^{\text {flex2/flex2 }}$; blue curve; $T_{50}=40$ days; $\left.P<0.0001\right)$ and

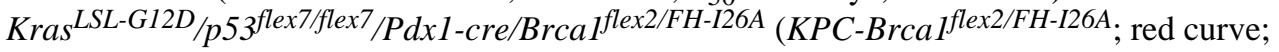
$T_{50}=65$ days; $\left.P=0.2595\right)$ mice. (B) Kaplan-Meier survival curves of Brcal ${ }^{\text {flex2/flex } 2 /}$ Wap $^{\text {crel+ }}\left(C\right.$-Brcal ${ }^{\text {flex } 2 / f l e x 2}$; green curve; $T_{50}=512$ days $)$ females compared with

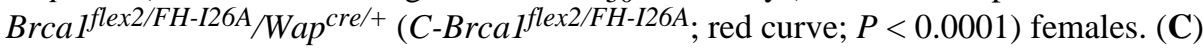
Kaplan-Meier survival curves of $p 53^{L S L-R 270 H /+} /$ Wap $^{\text {cre/+}}\left(\mathrm{PC}_{-} \mathrm{Brcal}^{+/+}\right.$; black curve; $T_{50}=$ 380 days) females compared with Brcal flex2/flex $2 / p 53^{L S L-R 270 H /+} /$ Wap $^{\text {cre } /+}(P C$ -

Brcal flex2/flex $;$ blue curve; $T_{50}=309$ days; $\left.P<0.0001\right)$ and Brcal flex $2 / F H-I 26 \mathrm{~A} /$

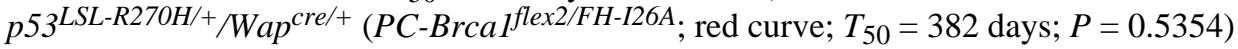

females. (D) Kaplan-Meier survival curves of control Brcal ${ }^{F H-W T / F H-W T}$ (black curve) mice compared with Brcal ${ }^{\text {tr/tr }}$ (blue curve; $T_{50}=529$ days; $P<0.0001$ ) and Brcal ${ }^{\text {FH-I26A/FH-I26A }}$ and Brcal $^{\mathrm{FH}-\mathrm{I2} 6 \mathrm{~A} / \mathrm{-}}$ (red curve; $P=0.5197$ ) mice. 
A

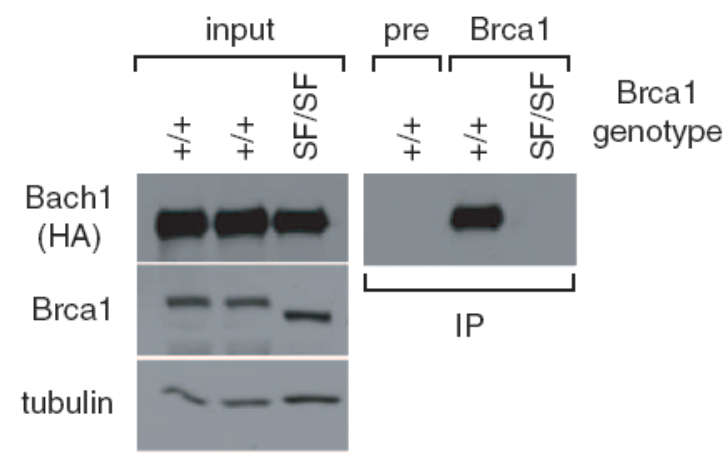

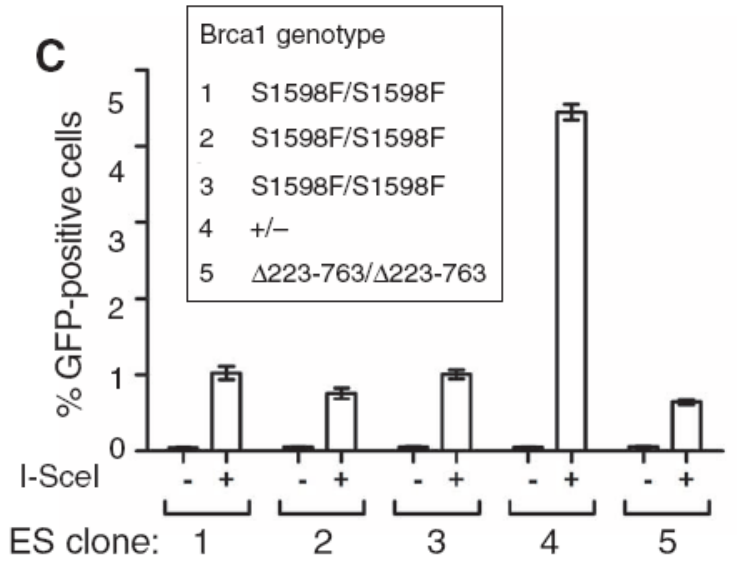

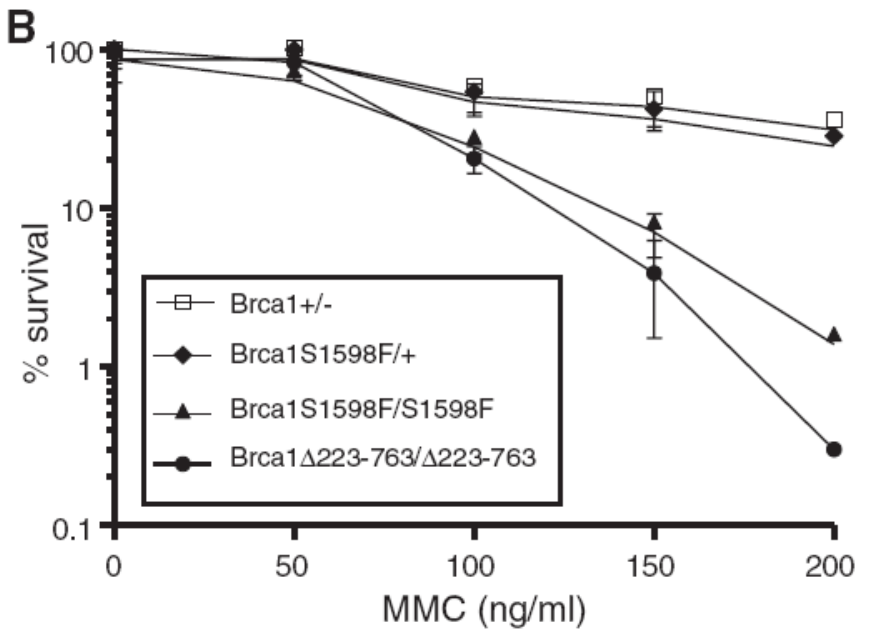

Fig. 2.

The BRCT phospho-recognition property of Brcal is critical for the DNA damage response. (A) S1598F ablates the interaction between Bach1/FancJ and endogenous Brca1. MEFs were prepared from mice with a knock-in allele $\left(B a c h 1^{W T-F H}\right)$ encoding wild-type Bach1 with C-terminal Flag-hemagglutinin (HA) epitopes. Bach1WT-FH and Brca1 levels were examined by immunoblotting lysates of $\mathrm{BrCal}^{+/+} / \mathrm{Bachl}^{\mathrm{WT}-\mathrm{FH} /+}$ and $\mathrm{BrCal}^{\text {S1598F/S1598F/ }}$ $B a c h 1^{W T-F H /+}$ MEFs (left). To evaluate the Brca1/Bach $1^{\text {WT-FH }}$ interaction, lysates were immunoprecipitated with a Brca1-specific antiserum or corresponding preimmune serum (pre) and immunoblotted with HA-specific antibodies (right). The input (left) represents $2.5 \%$ of the protein amount used for immunoprecipitation (right). (B) Brcal $^{\text {S1598F/S1598F }}$ cells are sensitive to genotoxic stress. ES cells proficient $\left(\mathrm{BrCal}^{+/-}\right.$and $\mathrm{BrCal}^{\mathrm{S1598F/+}}$ ) or deficient (Brcal ${ }^{S 1598 F / S 1598 F}$ ) for BRCT phospho-recognition were examined for mitomycin $\mathrm{C}$ (MMC) sensitivity in clonogenic survival assays, together with ES cells homozygous for the hypomorphic Brcal ${ }^{\Delta 223-763}$ mutation. (C) BRCT phospho-recognition by Brca1 is essential for homology-directed DNA repair. BrCal ${ }^{+/-}$, BrCal $^{S 1598 F / S 1598 F}$, and $B r c a 1^{\Delta 223-763 / \Delta 223-763}$ ES subclones containing the direct repeat-green fluorescent protein (DR-GFP) substrate integrated into the Piml locus were transfected with either an I-SceI expression vector or empty vector. I-SceI strongly induced the number of GFP-positive cells in $\mathrm{BrCal}^{+/-}$cells (clone 4), indicating efficient homology-directed repair, but not in Brcal ${ }^{S 1598 F / S 1598 F}$ cells (clones 1 to 3). The reduction in GFP-positive cells in Brcal $^{S 1598 F / S 1598 F}$ cells was similar to that of Brcal ${ }^{\Delta 223-763 / \Delta 223-763}$ ES cells (clone 5), which are known to be deficient in homology-directed repair (28). 

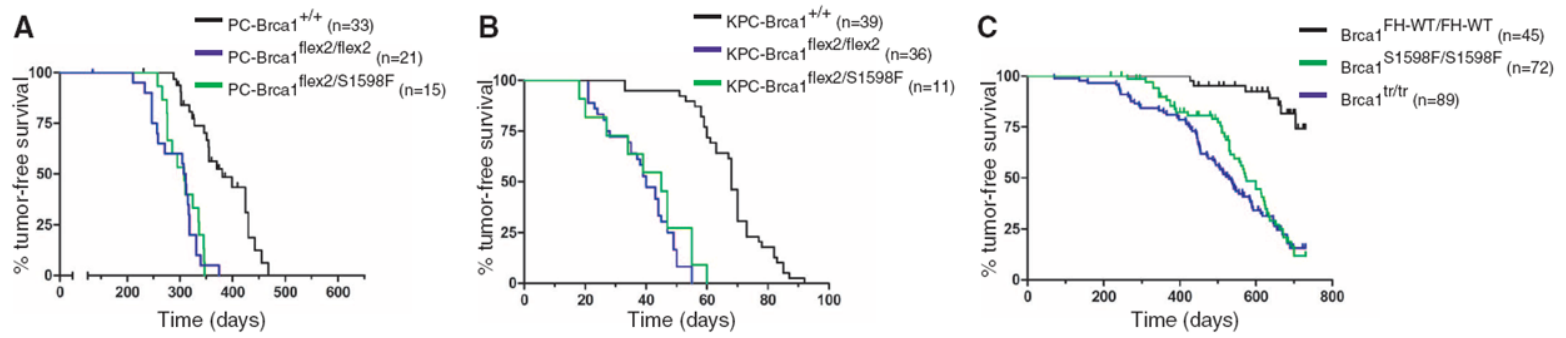

Fig. 3.

BRCT phospho-recognition is essential for Brca1 tumor suppression. (A) Kaplan-Meier tumor-free survival curves of $p 53^{L S L-R 270 H /+} / \mathrm{Wap}^{\mathrm{cre} /+}\left(\mathrm{PC}-\mathrm{Brcal}^{+/+}\right.$; black curve; $T_{50}=$ 380 days) females compared with Brcal flex2/flex $/ p 53^{L S L-R 270 H /+} / \mathrm{Wap}^{\mathrm{cre} /+}(\mathrm{PC}$ -

Brcal flex2/flex2 ; blue curve; $T_{50}=309$ days; $\left.P<0.0001\right)$ and Brcal flex2/S1598F/

p53 $3^{L S L-R 270 H /+} /$ Wap $^{\text {cre/+ }}\left(P C-B r c a l^{\text {flex } 2 / S 1598 F}\right.$; green curve; $T_{50}=308$ days; $\left.P<0.0001\right)$ females. (B) Kaplan-Meier survival curves of $\mathrm{Kras}^{L S L-G 12 D} / \mathrm{p}^{2} 3_{\text {flex } 7 / f l e x} 7 / P d x 1$-cre $(K P C$ -

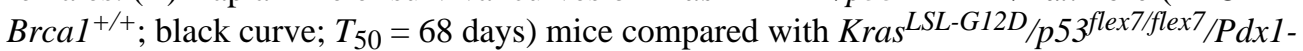
cre/Brcal $^{\text {flex2/flex2 }}$ (KPC-Brcal ${ }^{\text {flex2/flex2 }}$; blue curve; $T_{50}=40$ days; $\left.P<0.0001\right)$ and Kras $^{L S L-G 12 D / p 53^{\text {flex7/flex7} / P d x l-c r e / B r c a l ~ f l e x ~} 2 / S 1598 F}$ (KPC-Brcalflex2/S1598F ;reen curve; $T_{50}=45$ days; $P<0.0001$ ) mice. $(\mathbf{C})$ Kaplan-Meier survival curves of Brcal ${ }^{F H-W T / F H-W T}$ (black curve) mice compared with Brcal ${ }^{\text {tr/tr }}$ (blue curve; $P<0.0001$ ) and Brcal $^{\text {S1598F/S1598F }}$ (green curve; $P<0.0001$ ) mice. 